\title{
Network Pharmacology-Based Investigation of the Mechanism of Action of Plantaginis Herba in Hyperuricemia Treatment
}

\author{
Rong Tang $\mathbb{D},,^{1}$ Xiaoqing Peng $\mathbb{D},{ }^{1}$ Yan Wang $\mathbb{D},,^{1}$ Xiaohong Zhou $\mathbb{D}^{1},{ }^{1}$ and Hong Liu $\mathbb{D}^{2}$ \\ ${ }^{1}$ Department of Pharmacy, Guangzhou First People's Hospital, School of Medicine, South China University of Technology, \\ Panfu Road 1, Guangzhou 510013, Guangdong, China \\ ${ }^{2}$ Department of Traditional Chinese Medicine, The First Affiliated Hospital of Guangdong Pharmaceutical University, \\ Gonghexiheng Street 1, Guangzhou 510080, Guangdong, China \\ Correspondence should be addressed to Hong Liu; 303280010@qq.com
}

Received 27 January 2021; Revised 21 March 2021; Accepted 29 March 2021; Published 9 April 2021

Academic Editor: Amin Tamadon

Copyright ( $\odot 2021$ Rong Tang et al. This is an open access article distributed under the Creative Commons Attribution License, which permits unrestricted use, distribution, and reproduction in any medium, provided the original work is properly cited.

\begin{abstract}
This study used a network pharmacology approach to investigate the potential active ingredients of Plantaginis Herba and its underlying mechanisms in hyperuricemia treatment. The potential active ingredients of Plantaginis Herba were obtained from TCMSP and ETCM databases, and the potential targets of the active ingredients were predicted using the Swiss TargetPrediction database. The potential therapeutic targets of hyperuricemia were retrieved from the GeneCards, DisGeNET, and Online Mendelian Inheritance in Man (OMIM) databases. Then, the integrative bioinformatics analyses of candidates were performed by GO analysis, KEGG analysis, and PPI network construction. There were 15 predicted active ingredients in Plantaginis Herba and 41 common targets that may be involved in the treatment of hyperuricemia. A total of $61 \mathrm{GO}$ annotations and 35 signaling pathways were identified by enrichment analysis $(P<0.01)$. The underlying mechanisms of Plantaginis Herba may be related to insulin resistance, PI3K/AKT, TNF, VEGF, AMPK, and glucagon signaling pathways. Thus, the present study provided potential and promising strategies of Plantaginis Herba for hyperuricemia treatment.
\end{abstract}

\section{Introduction}

Hyperuricemia is a syndrome involving metabolic abnormalities (increases in uric acid production and/or decreased excretion of uric acid) caused by purine metabolism disorders [1]. Excessive uric acid in blood can lead to the formation of sodium urate crystals, which are then deposited in joints, thus inducing gout [2-4].

The pathogenesis of hyperuricemia is related to a variety of factors, such as genetics, intense exercise, alcoholism, radiotherapy and chemotherapy, renal insufficiency, drugs, and metabolic syndrome [5]. In recent years, the prevalence of hyperuricemia has significantly increased and has shown a trend of younger age of onset $[6,7]$. In China, the prevalence of hyperuricemia is $13.3 \%$, indicating that it has become another common metabolic disease, such as diabetes [8].

Currently, to treat hyperuricemia, Western medicine therapeutic strategies mainly involve the inhibition of uric acid synthesis, the promotion of uric acid excretion, and the alkalinization of urine. The commonly used Western medicines are allopurinol and benzbromarone, which can cause adverse reactions in the liver, gastrointestinal tract, and on the skin [2].

Plantaginis Herba is the dried whole grass of Plantago asiatica L. or Plantago depressa Willd, which belongs to Plantaginaceae. It can help reduce fever and diuresis, dispel phlegm, and detoxify [9]. Previous studies have shown that Plantaginis Herba reduces uric acid, which may be achieved through inhibiting the activities of xanthine oxidase (XOD) and adenosine deaminase (ADA) and downregulating the mRNA expression of renal urate transporter 1 (mURAT1) [10]. However, it is difficult to compressively explain the synergism of multi-ingredient/multitarget/multipathway of traditional Chinese medicine (TCM) through single-target or single-pathway studies. In the present study, we utilized TCM network pharmacology to systematically analyze the 
effective active ingredients and potential targets of Plantaginis Herba to explore its possible mechanism.

\section{Materials and Methods}

2.1. Prediction of the Active Ingredients of Plantaginis Herba and Its Targets. The keyword "Plantaginis Herba" was searched in the Chinese medicine systems pharmacology database and analysis platform (TCMSP) (http://tcmspw. $\mathrm{com} /$ ) and the Encyclopedia of Traditional Chinese Medicine (ETCM) database (http://www.tcmip.cn/ETCM/), and the retrieved results were screened based on the following parameters: oral bioavailability $(\mathrm{OB}) \geq 30 \%$ and drug-likeness $(\mathrm{DL}) \geq 0.18$. Then, the existing literature was searched for studies involving the ingredients of Plantaginis Herba for hyperuricemia treatment. The molecular structures (SDF format) of the obtained ingredients were obtained from the PubChem database (https://pubchem.ncbi.nlm.nih.gov/), and potential targets were predicted using the Swiss TargetPrediction database (http://www.swisstargetprediction. $\mathrm{ch} /$ ). The potential targets were subjected to gene normalization using the UniProt database (http://www.UniProt. org/). The active ingredients and corresponding targets of Plantaginis Herba were imported into Cytoscape 3.8.0 software to construct an active ingredient-target network for Plantaginis Herba.

\subsection{Prediction of Hyperuricemia-Related Targets.} "Hyperuricemia" and "hyperuricaemia" were used as keywords to search the following disease gene databases: DrugBank (https://www.drugbank.ca/), DisGeNET (https:// www.disgenet.org/), GeneCards (https://www.genecards. org/), Online Mendelian Inheritance in Man (OMIM) (https://www.omim.org/), and Therapeutic Target Database (TTD) (http://db.idrblab.net/ttd/). Additionally, using the data retrieved from DrugBank, targets of first-line Western drugs used for the clinical treatment of hyperuricemia were also included. The obtained target genes were imported into the UniProt database for gene standardization.

\subsection{Protein-Protein Interaction (PPI) Network Analysis.} The targets of the active ingredients of Plantaginis Herba and hyperuricemia-related targets were imported into Venny 2.1 software to generate a Venn diagram to obtain the common targets of Plantaginis Herba for the treatment of hyperuricemia. The common targets were imported into the STRING database (https://string-db.org/), and the species "Homo sapiens" was selected to obtain a PPI network. The data were then imported into Cytoscape 3.8.0 software for visualization, and the core targets in the PPI network were obtained.

2.4. GO Analysis and KEGG Pathway Enrichment Analysis. The overlapped targets were imported into DAVID 6.8 (https://david.ncifcrf.gov/) for Gene Ontology (GO) analysis and Kyoto Genomics and Genomics Encyclopedia (KEGG) analysis; the screening parameter was set as the false discovery rate $(F D R)<0.05$. The results were visualized using Image GP, and the analyzed results were displayed using bubble diagrams.

2.5. Construction of the Target-Pathway Network. Targets enriched in the pathways associated with hyperuricemia were ascertained, and the active ingredients acting on these targets were identified. The interaction among the active ingredients, targets, and pathways was established using nodes to represent the active ingredients, targets, and pathways and edges to represent the association between a certain target and a certain pathway or the action of an active ingredient on a certain target. The file data were imported into Cytoscape 3.8.0 to construct an "active ingredienttarget-pathway" network, and the topological attributes of the nodes in this network were analyzed to clarify the material basis and multi-ingredient, multitarget, and multipathway molecular mechanisms for the treatment of hyperuricemia with Plantaginis Herba.

\section{Results}

3.1. The Candidate Ingredients of Plantaginis Herba and Its Targets. We firstly screened the active ingredients of Plantaginis Herba and predicted their relative targets. Based on the $\mathrm{OB}$ and DL parameters, 10 active ingredients of Plantaginis Herba were obtained from the TCMSP and ETCM databases. In addition, 5 reported active ingredients of Plantaginis Herba, such as verbascoside, eupatilin, nepetin, jaceosidin, and eupatorin, were also included $[11,12]$. Therefore, a total of 15 active ingredients were finally obtained (Table 1). A total of 232 targets were obtained by importing the identified active ingredients into the PubChem website and the Swiss TargetPrediction database. Cytoscape 3.8.0 software was used to visualize and analyze the networks for 15 active ingredients and 232 targets of Plantaginis Herba, and the following core ingredients were identified: hispidulin, baicalein, luteolin, 6-hydroxyluteolin, eupatilin, nepetin, jaceosidin, and eupatorin (Figure 1).

3.2. Construction of the PPI Network of the Active Ingredients of Plantaginis Herba and Hyperuricemia. Hyperuricemia-related targets were searched and retrieved from 5 disease databases: DrugBank, DisGeNET, GeneCards, OMIM, and TTD; 65, 196, 668, 6, and 0 targets were retrieved, respectively. After assessing the above targets and removing duplicate targets, we finally obtained 768 disease targets. Forty-one common targets were obtained by comparing the targets obtained above (Figure 2). These common targets were imputed into STRING to construct a PPI network, and the network was visualized using Cytoscape 3.8.0 (Figure 3). The PPI network consisted of 41 nodes with 188 edges, with an average nodal degree of 9.17 and an average compactness value of 0.584 . Targets with values not less than the average values of node degree and compactness were used as core targets. The core targets of Plantaginis Herba for the treatment of hyperuricemia were AKT1, mitogen-activated protein kinase 3 (MAPK3), MAPK1, 
TABle 1: Active ingredients of Plantaginis Herba.

\begin{tabular}{lccccc}
\hline No. & Mol ID & Mol name & ID & OB (\%) & DL \\
\hline 1 & MOL001735 & Dinatin & CQC1 & 30.97 & 0.27 \\
2 & MOL002714 & Baicalein & CQC2 & 33.52 & 0.21 \\
3 & MOL002776 & Baicalin & CQC3 & 40.12 & 0.75 \\
4 & MOL000359 & Sitosterol & CQC4 & 36.91 & 0.75 \\
5 & MOL004004 & 6-OH-Luteolin & CQC5 & 46.93 & 0.28 \\
6 & MOL000449 & Stigmasterol & CQC6 & 43.83 & 0.76 \\
7 & MOL000006 & Luteolin & CQC7 & 36.16 & 0.25 \\
8 & MOL007783 & Melampyroside & CQC8 & 57.5 & 0.8 \\
9 & MOL007796 & Stigmasterol & CQC9 & 38.09 & 0.4 \\
10 & MOL007799 & p-Sitosterol palmitate & CQC10 & 30.91 & 0.4 \\
11 & MOL003333 & Acteoside & CQC11 & 2.94 & 0.62 \\
12 & MOL005734 & Eupatilin & CQC12 & 29.39 & 0.38 \\
13 & MOL005305 & Nepetin & CQC13 & 26.75 & 0.31 \\
14 & MOL009297 & Jaceosidin & CQC14 & 2.14 & 0.34 \\
15 & MOL001733 & Eupatorin & CQC15 & 30.23 & 0.37 \\
\hline
\end{tabular}

tumor necrosis factor (TNF), prostaglandin-endoperoxide synthase 2 (PTGS2), sirtuin 1 (SIRT1), estrogen receptor 1 (ESR1), and androgen receptor (AR).

3.3. GO Analysis and KEGG Pathway Enrichment Analysis of Hub Targets. The common targets were enriched in $61 \mathrm{GO}$ functions $(P<0.01$, Figure 4$)$. Enrichment was observed in the following molecular functions $(n=16)$ : enzyme binding, protein binding, steroid receptor activity, transcription factor binding, ATP binding, and RNA polymerase II transcription factor activity. Enrichment was observed in the following biological processes: the positive regulation of RNA polymerase II promoter transcription, smooth muscle cell proliferation, positive regulation of drug reactions, nitric oxide biosynthesis, cell proliferation, negative regulation of gene expression, lipopolysaccharide-mediated signaling pathways, and steroid-mediated signaling pathways. A total of 35 KEGG pathways were enriched $(P<0.01$, Figure 5$)$. Among those enriched, the signaling pathways associated with hyperuricemia include the PI3K/AKT, TNF, VEGF, AMPK, insulin resistance, and glucagon signaling pathways, suggesting that Plantaginis Herba may play a role in treating hyperuricemia mainly through the regulation of these 6 signaling pathways.

3.4. Active Ingredient-Target-Pathway Network Analysis. An "active ingredient-target-pathway" network was constructed based on the 6 most relevant signaling pathways obtained in Section 2.4 (Figure 6). There were 39 nodes (including 14 active ingredients, 19 targets, and 6 pathways) and 108 edges in the network. In this network, the active ingredients with more targets were baicalein, luteolin, eupatilin, and 6-hydroxyluteolin, suggesting that these ingredients may be the material basis through which Plantaginis Herba treats hyperuricemia. PTGS2, AKT1, IGF1R, TNF, KDR, PYGL, MAPK3, and MAPK1 were the targets that connected with more active ingredients and pathways, suggesting that these targets might be the key targets for the treatment of hyperuricemia with Plantaginis Herba; these results are consistent with the PPI network analysis results in Section 2.3. Therefore, active ingredients such as luteolin, eupatilin, 6-hydroxyluteolin, hispidulin, and baicalein act through targets such as PTGS2, AKT1, IGF1R, TNF, KDR, PYGL, MAPK3, and MAPK1 to jointly regulate signaling pathways such as PI3K/AKT, TNF, VEGF, AMPK, insulin resistance, and glucagon pathways to achieve hyperuricemia treatment efficacy.

\section{Discussion}

Plantaginis Herba clears heat, disinhibits dampness, increases diuresis, frees stranguries, eliminates phlegm, cools blood, and detoxifies [13, 14]. Modern pharmacological studies have also confirmed that the Plantaginis Herba extract can effectively treat hyperuricemia [15]. Previous studies have shown that a Chinese herbal compound containing S. moellendorffi, Smilacis glabrae Rhizoma, and Plantaginis Semen, at a ratio of $3: 1: 1$, can significantly inhibit the activation of nuclear factor $-\kappa \mathrm{B}(\mathrm{NF}-\kappa \mathrm{B})$ and the expression of its target genes, interleukin-1 $\beta$ (IL-1 $\beta$ ), prostaglandin E2 (PGE2), and IL-8, thereby playing an important role in the prevention and treatment of hyperuricemia and gout [16]. However, the effective and important active ingredients in this compound are not known. Other studies showed that verbascoside and 1-hydroxyindole-3-carbaldehyde, the extracts of Plantaginis Herba, significantly inhibited XOD activity and had significant therapeutic effects when used to treat hyperuricemia and gout [17]. In addition, active ingredients such as baicalein, baicalin, sitosterol, 6-hydroxyluteolin, stigmasterol, luteolin, and nepetin extracted from other herbs have been shown to significantly reduce uric acid levels [17-23]. In the present study, 15 active ingredients of Plantaginis Herba were obtained from the TCMSP and ETCM databases. However, the roles of hispidulin, melampyroside, stigmasterol palmitate, $\beta$-sitosterol palmitate, eupatilin, jaceosidin, and eupatorin in the treatment of hyperuricemia are still unclear. Therefore, the active ingredients of Plantaginis Herba identified in this study may play important roles in the prevention and treatment of hyperuricemia.

We constructed an "active ingredient-target-pathway" network to further investigate the mechanism of action underlying Plantaginis Herba and its active ingredients in the prevention and treatment of hyperuricemia. AKT1, MAPK3 (ERK1), MAPK1 (ERK2), TNF, PTGS2 (COX-2), KDR, PYGL, and IGF1R were key targets based on a topological data analysis of the network.

Accumulating previous studies have shown that AKT plays an important role in the occurrence and development of hyperuricemia and gout. Activation of the AMP-activated protein kinase (AMPK)/AKT/cAMP-response elementbinding protein (CREB) signaling axis promotes the binding of CREB to the ATP-binding cassette superfamily $\mathrm{G}$ member 2 (ABCG2) promoter and upregulates the expression of ABCG2, thereby promoting the excretion of uric acid [24]. Uric acid can downregulate the expression of ABCG2 by inhibiting the activation of AKT, leading to the intracellular accumulation of uric acid [25]. Interestingly, soluble uric 


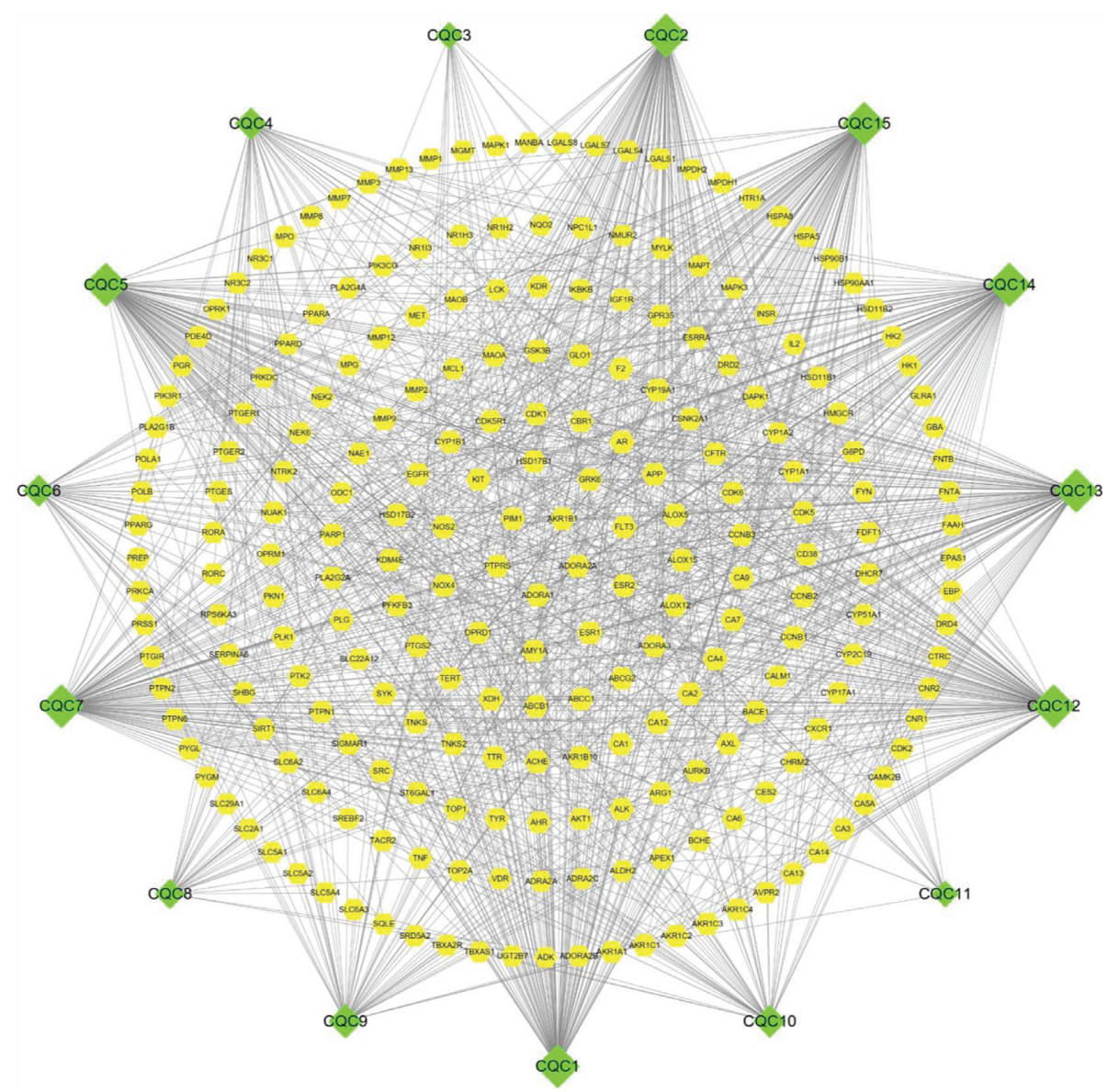

Figure 1: The active ingredient-target network for Plantaginis Herba. The rhombuses represent ingredient nodes, the hexagons represent predicted targets, and the connecting lines represent the interaction between the ingredients and the targets. The node size represents the corresponding degree value; a larger degree value corresponds to a larger node area, indicating that the node is more useful in the network.

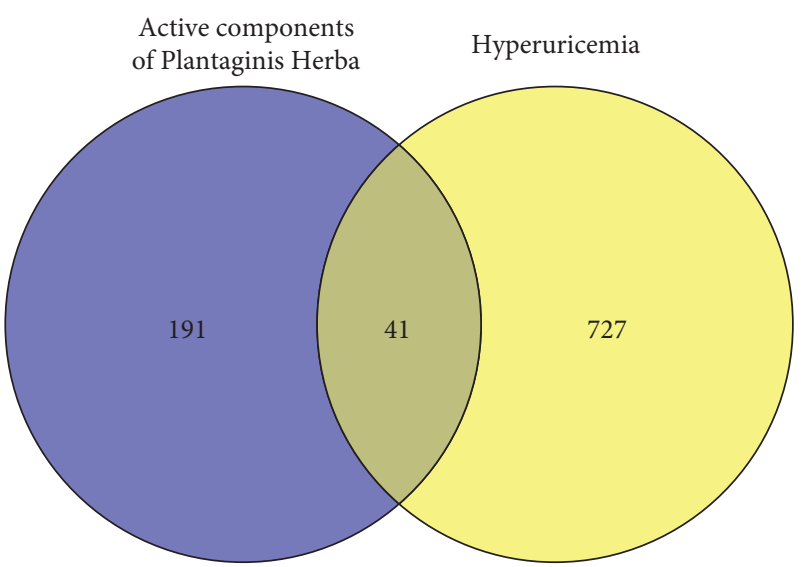

Figure 2: Venn diagram of targets of active ingredients of Plantaginis Herba and targets related to hyperuricemia.

acid activates the toll-like receptor 4 (TLR4)-NLR family pyrin domain containing 3 (NLRP3) and PI3K/AKT pathways and promotes the transcription of ABCG2 by upregulating PDZ domain containing 1 (PDZK1), thereby promoting the excretion of uric acid in the intestines, along with feces [26]. Other studies have shown that uric acid promotes the abnormal proliferation of glomerular mesangial cells through the activation of the nicotinamide adenine dinucleotide phosphate (NADPH)/ROS/extracellular receptor kinase 1/2 (ERK1/2) pathway, leading to glomerular injury [27]. Uric acid can upregulate the expression of TNF- $\alpha$ through the ROS/MAPK (p38)/NF- $\kappa$ B 


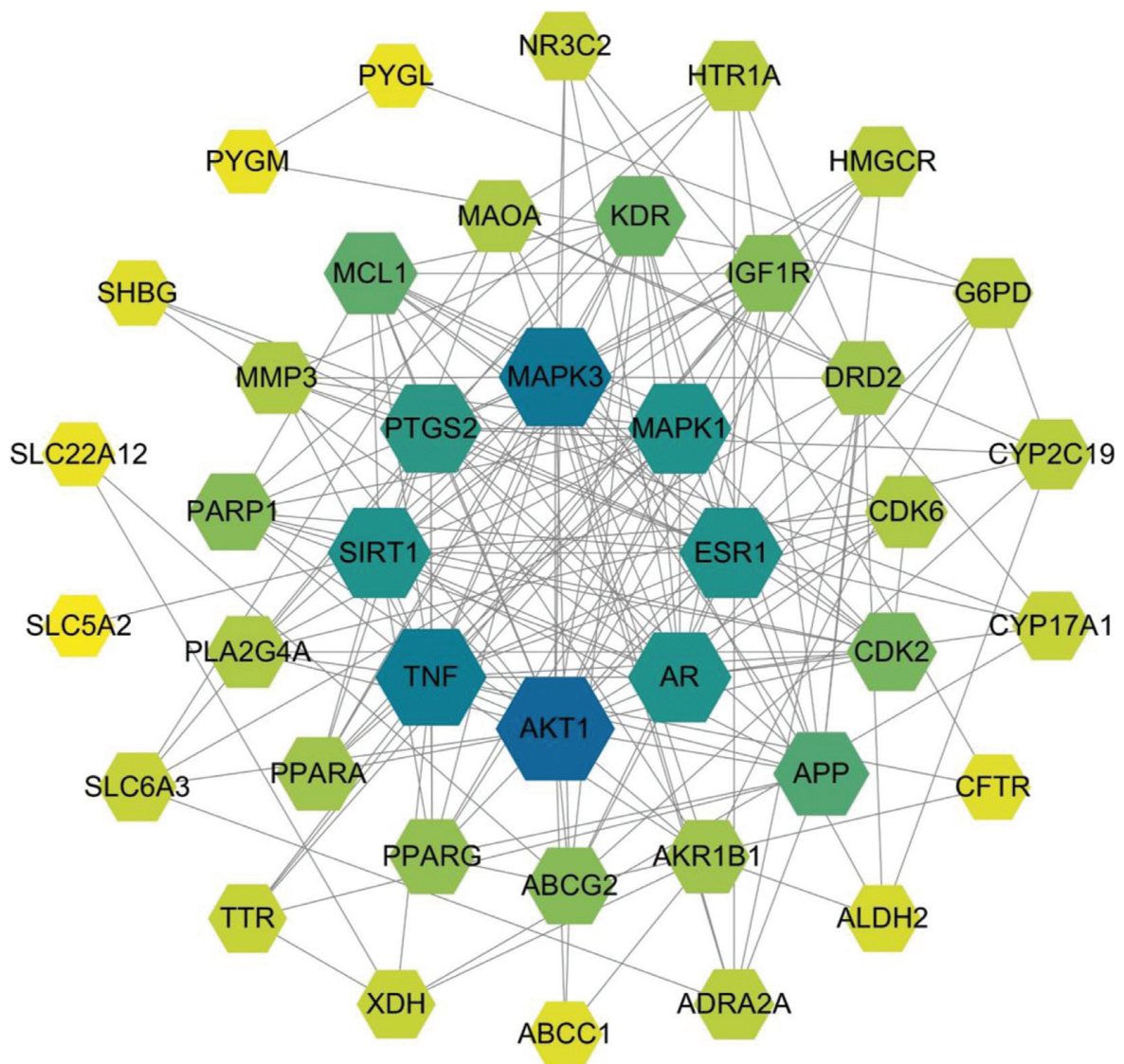

FIgURE 3: PPI network of the common targets of active ingredients of Plantaginis Herba and hyperuricemia.

GO:0042802 identical protein binding GO:0005524 ATP binding GO:0019899 enzyme binding GO:0042803 protein homodimerization activity GO:0008134 transcription factor binding GO:0003707 steroid hormone receptor activity GO:0008144 drug binding GO:0005496 steroid binding GO:0004879 RNA polymerase II transcription factor activity, ligand-activated sequence-specific DNA binding
GO:0051721 protein phosphatase $2 \mathrm{~A}$ binding GO:0005829 cytosol GO:0005654 nucleoplasm GO:0070062 extracellular exosome

GO:0005887 integral component of plasma membrane GO:0043234 protein complex GO:0030424 axon

GO:0043235 receptor complex GO:0005901 caveola GO:0045944 positive regulation of transcription from RNA polymerase II promoter GO :0045893 positive regulation of cell proliferation templated GO:0048661 positive GO:0042493 response to drug GO:0001934 positive regulation of protein phosphorylation GO:0010629 negative regulation of gene expression GO:0031663 Iipopolysaccharide-mediated signaling pathway GO:0045429 positive regulation of nitric oxide biosynthetic GO:0043401 steroid hormone-mediated signaling pathway

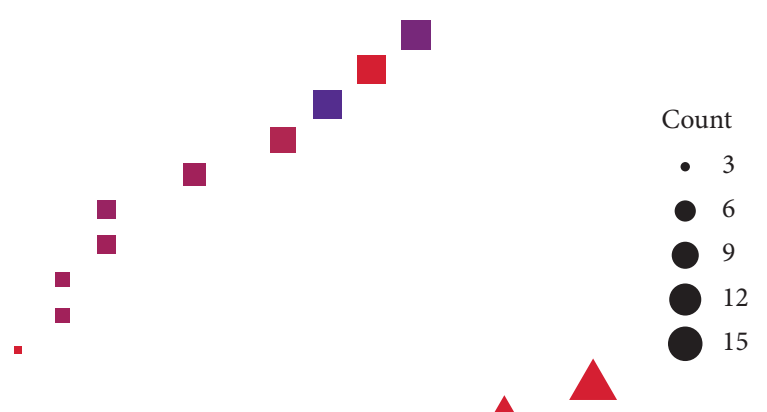

neglog10_Pvalue

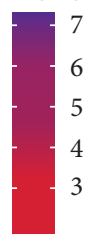

\footnotetext{
- GOTERM_BP_DIRECT

- GOTERM_CC_DIRECT
}

SampleGroup

Figure 4: Bubble diagram of GO enrichment of common targets. 


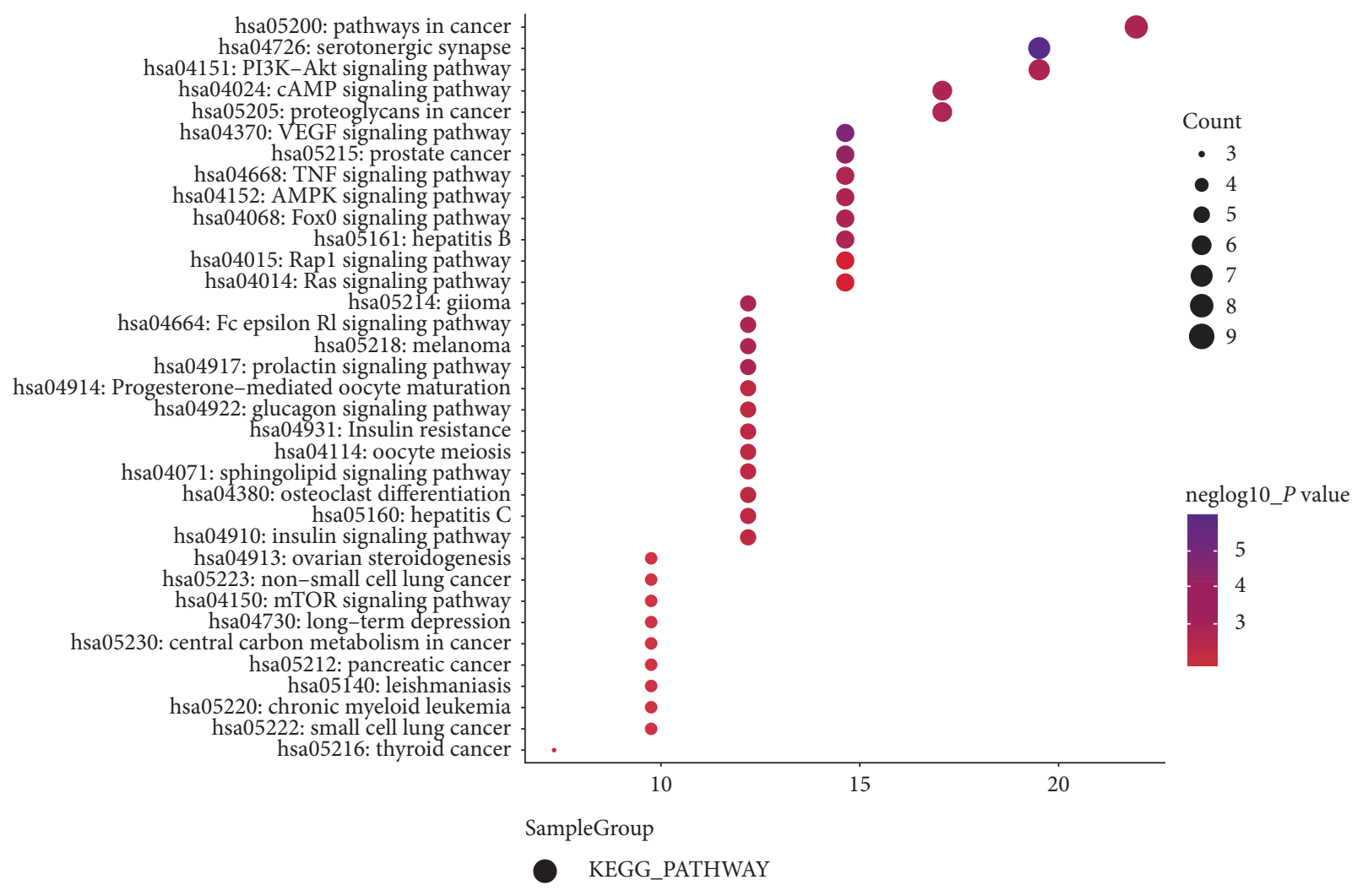

FIGURE 5: KEGG pathway analysis of common targets.

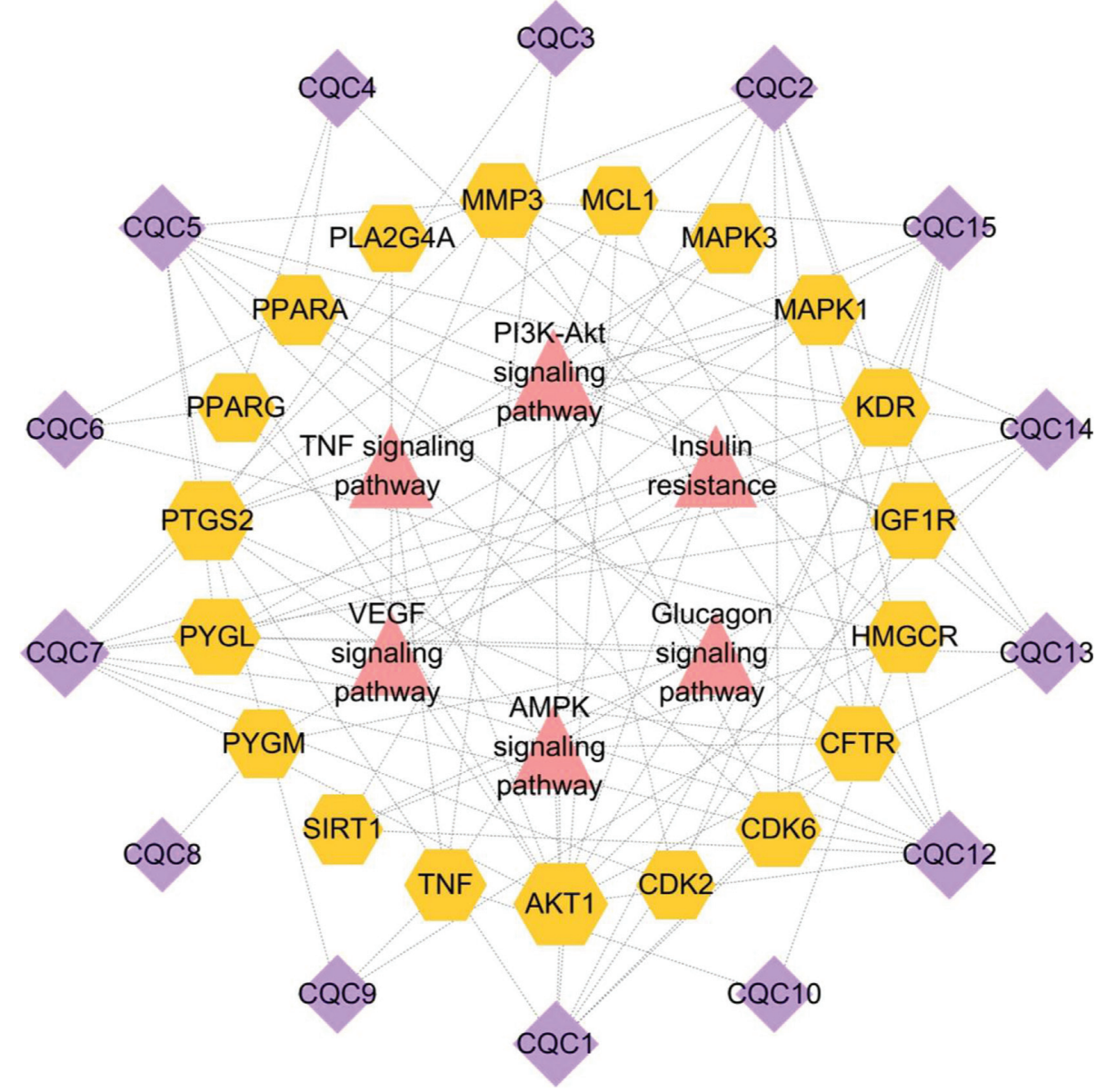

Figure 6: Active ingredient-target-pathway network. A triangle represents a signaling pathway, a hexagon represents a target, and a diamond represents an active ingredient. A node with a larger size indicates having more connected compounds or targets or pathways. 
signaling axis, thereby promoting the inflammation and necrosis of vascular smooth muscle cells [28]. Uric acid promotes the activation of platelet-derived growth factor receptor- $\beta$ (PDGFR- $\beta$ ) through the p38 MAPK and ERK1/2 pathways, thereby inducing and aggravating cardiovascular diseases [29]. In a hyperuricemia animal model, uric acid can upregulate the expression of inflammatory factors such as cyclooxygenase-2 (COX-2), IL- $1 \beta$, and TNF- $\alpha$ and play an important role in the development of complications related to hyperuricemia [30].

Other studies have shown that plantamajoside, an extract of Plantaginis Herba, can inhibit the activation of histone deacetylase 2 (HDAC2), and the transduction of downstream signals, AKT/GSK-3 $\beta$, in turn has an important protective effect against cardiac hypertrophy [31]. Ethanol extracts of Plantaginis Herba inhibit AKT phosphorylation but can alleviate the deterioration of diabetic retinopathy through the inhibition of inflammatory signals [32]. The regulatory effects of the active ingredients of Plantaginis Herba selected in this study on AKT and their roles in the prevention and treatment of hyperuricemia and gout are still unclear and have not been reported. In addition, few studies have reported the regulatory effects of the active ingredients selected in this study on other target genes. Therefore, it is particularly important to further study the exact mechanism of action of these active ingredients.

In summary, this study selected a group of active ingredients of Plantaginis Herba with potential application prospects. They may promote ABCG2 expression and uric acid excretion through the activation of AKT-related pathways. Additionally, these active ingredients may also downregulate the body's inflammatory response by inhibiting the expression of the inflammatory factors COX2 and TNF- $\alpha$ and inhibiting the activation of ERK1/2 and the abnormal proliferation of glomerular mesangial cells. These active ingredients may alleviate hyperuricemia, slow the occurrence and development of gout, and inhibit the damage caused by high uric acid levels in the kidney and cardiovascular system through the integration of multiple signals. Thus, the present study suggests a potential mechanism and potential clinical application values of Plantaginis Herba for the treatment of hyperuricemia and its complications.

\section{Data Availability}

The data used to support the findings of this study are available from the corresponding author upon request.

\section{Disclosure}

All funders had no role in the design of the study and collection, analysis, interpretation of data, writing the manuscript, and the decision to submit the manuscript for publication.

\section{Conflicts of Interest}

The authors declare no conflicts of interest.

\section{Authors' Contributions}

Hong Liu conceived and designed the study and submitted the manuscript. Rong Tang, Xiaoqing Peng, Yan Wan, and Xiaohong Zhou acquired and analyzed the data. Rong Tang prepared the manuscript. All authors read and approved the final manuscript.

\section{Acknowledgments}

This work was supported by Basic and Applied Basic Research Fund Project of Guangdong Province (2020A1515110615) and the Medical Research Foundation of Guangdong Province (B2020043).

\section{References}

[1] J. G. Puig and M. A. Martínez, "Hyperuricemia, gout and the metabolic syndrome," Current Opinion In Rheumatology, vol. 20, no. 2, pp. 187-191, 2008.

[2] C. S. O. Endocrinology, "Guideline for the diagnosis and management of hyperuricemia and gout in China (2019)," Chinese Journal of Endocrinology And Metabolism, vol. 36, pp. 1-13, 2020.

[3] M. Skoczyńska, M. Chowaniec, A. Szymczak, A. LangnerHetmańczuk, B. Maciążek-Chyra, and P. Wiland, "Pathophysiology of hyperuricemia and its clinical significance - a narrative review," Reumatologia, vol. 58, no. 5, pp. 312-323, 2020.

[4] M. P. Leask, N. A. Sumpter, A. S. Lupi et al., "The shared genetic basis of hyperuricemia, gout, and kidney function," Seminars In Nephrology, vol. 40, no. 6, pp. 586-599, 2020.

[5] L. Li, Y. Zhang, and C. Zeng, "Update on the epidemiology, genetics, and therapeutic options of hyperuricemia," American Journal of Translational Research, vol. 12, no. 7, pp. 3167-3181, 2020.

[6] J. Huang, Z. F. Ma, Y. Zhang et al., "Geographical distribution of hyperuricemia in mainland China: a comprehensive systematic review and meta-analysis," Global Health Research And Policy, vol. 5, p. 52, 2020.

[7] H. Zhou, Z. F. Ma, Y. Lu et al., "Elevated serum uric acid, hyperuricaemia and dietary patterns among adolescents in mainland China," Journal of Pediatric Endocrinology and Metabolism, vol. 33, no. 4, pp. 487-493, 2020.

[8] R. Liu, C. Han, D. Wu et al., "Prevalence of hyperuricemia and gout in mainland China from 2000 to 2014: a systematic review and meta-analysis," Biomed Research International, vol. 2015, Article ID 762820, 12 pages, 2015.

[9] Y. Liu, Z. Wang, and J. Zhang, "Plantago asiatica L. Cheqian (cheqian, Asiatic plantain),” pp. 749-756, 2015.

[10] J. Zeng, J. Wei, Z. YY, J. Zhu, X. Wang, and G. Luo, "The research of Plantago asiatica L. Herbs extracts reduce the level of uric acid in hyperuricemia mice and it's mechanism," Lishizhen Medicine and Materia Medica Research, vol. 24, pp. 2064-2066, 2013.

[11] J. Wang, B. Xu, J. Zeng et al., "Development of an electron transfer channel blocking method for screening of xanthine oxidase inhibitors from Plantago asiatica L," Chinese Journal of Analytical Chemistry, vol. 36, pp. 1816-1822, 2016.

[12] M. Li, Y. Yu, J. Liu, Z. Chen, and S. Cao, "Investigation of the interaction between benzaldehyde thiosemicarbazone compounds and xanthine oxidase," Journal of Molecular Structure, vol. 1159, pp. 23-32, 2018. 
[13] X. Yang, M. Y. Li, C. Y. Yan et al., "[Research progress on chemical composition and pharmacological effects of periplocae cortex and predictive analysis on Q-marker]," Zhongguo Zhong Yao Za Zhi, vol. 45, pp. 2772-2783, 2020.

[14] Membership of The Pharmacopoeia Commission of The People's Republic of China, Pharmacopoeia of the People's Republic of China (Part I), p. 64, Chemical Industry Press, Beijing, China, 2010.

[15] Y. Qian, Q. C. FU, J. P. Wang, and H. B. Bai, "Study on extraction process of active ingredients with hypouricuria effect from Plantaginis Herba," Journal of Zhejiang University (Science Edition), vol. 37, pp. 560-562, 2010.

[16] X. Y. Zhang, J. Cheng, P. Zhao, K. L. Chen, and J. Li, "Screening the best compatibility of selaginella moellendorffii prescription on hyperuricemia and gouty arthritis and its mechanism," Evidence-Based Complementary and Alternative Medicine, vol. 2019, Article ID 7263034, 10 pages, 2019.

[17] J. X. Zeng, J. Wang, S. W. Zhang et al., "Antigout effects of Plantago asiatica: xanthine oxidase inhibitory activities assessed by electrochemical biosensing method," EvidenceBased Complementary and Alternative Medicine, vol. 2018, Article ID 1364617, 11 pages, 2018.

[18] F. Rozza, V. Trimarco, R. Izzo, D. Grassi, and C. Ferri, "Effects of a novel fixed combination of nutraceuticals on serum uric acid concentrations and the lipid profile in asymptomatic hyperuricemic patients," High Blood Pressure \& Cardiovascular Prevention, vol. 23, no. 4, pp. 381-386, 2016.

[19] X. Meng, Z. Mao, X. Li et al., "Baicalein decreases uric acid and prevents hyperuricemic nephropathy in mice," Oncotarget, vol. 8, no. 25, pp. 40305-40317, 2017.

[20] Z. Ferraz-Filha, M. Michel Araújo, F. Ferrari, I. Dutra, and D. Saúde-Guimarães, "Tabebuia roseoalba: in vivo hypouricemic and anti-inflammatory effects of its ethanolic extract and constituents," Planta Medica, vol. 82, no. 16, pp. 1395-1402, 2016.

[21] L.-C. Cheng, V. Murugaiyah, and K.-L. Chan, "Flavonoids and phenylethanoid glycosides from lippia nodiflora as promising antihyperuricemic agents and elucidation of their mechanism of action," Journal of Ethnopharmacology, vol. 176, pp. 485-493, 2015.

[22] Y. Ding, X. Shi, X. Shuai et al., "Luteolin prevents uric acidinduced pancreatic $\beta$-cell dysfunction," Journal of Biomedical Research, vol. 28, no. 4, pp. 292-298, 2014.

[23] J. Kim, W. Kim, J. Hyun et al., "Salvia plebeia extract inhibits xanthine oxidase activity in vitro and reduces serum uric acid in an animal model of hyperuricemia," Planta Medica, vol. 83, no. 17, pp. 1335-1341, 2017.

[24] Y.-h. Lu, Y.-p. Chang, T. Li et al., "Empagliflozin attenuates hyperuricemia by upregulation of ABCG2 via AMPK/AKT/ CREB signaling pathway in type 2 diabetic mice," International Journal Of Biological Sciences, vol. 16, no. 3, pp. 529$542,2020$.

[25] H. Komori, K. Yamada, and I. Tamai, "Hyperuricemia enhances intracellular urate accumulation via down-regulation of cell-surface BCRP/ABCG2 expression in vascular endothelial cells," Biochimica et Biophysica Acta (BBA) - Biomembranes, vol. 1860, no. 5, pp. 973-980, 2018.

[26] M. Chen, X. Lu, C. Lu et al., "Soluble uric acid increases PDZK1 and ABCG2 expression in human intestinal cell lines via the TLR4-NLRP3 inflammasome and PI3K/akt signaling pathway," Arthritis Research \& Therapy, vol. 20, p. 20, 2018.

[27] Y. Zhuang, Q. Feng, G. Ding et al., "Activation of ERK1/2 By NADPH oxidase-originated reactive oxygen species mediates uric acid-induced mesangial cell proliferation," American
Journal of Physiology-Renal Physiology, vol. 307, no. 4, pp. F396-F406, 2014.

[28] L. Tang, Y. e. Xu, Y. Wei, and X. He, "Uric acid induces the expression of TNF- $\alpha$ via the ROS-MAPK-NF- $\kappa$ B signaling pathway in rat vascular smooth muscle cells," Molecular Medicine Reports, vol. 16, no. 5, pp. 6928-6933, 2017.

[29] M. Kırça, N. Oğuz, A. Çetin, F. Uzuner, and A. Yeşilkaya, "Uric acid stimulates proliferative pathways in vascular smooth muscle cells through the activation of p38 MAPK, p44/42 MAPK and PDGFR $\beta$," Journal Of Receptor And Signal Transduction Research, vol. 37, no. 2, pp. 167-173, 2017.

[30] A. Mehmood, L. Zhao, M. Ishaq et al., "Anti-hyperuricemic potential of stevia (stevia rebaudiana bertoni) residue extract in hyperuricemic mice," Food \& Function, vol. 11, no. 7, pp. 6387-6406, 2020.

[31] L. Shang, L. Pin, S. Zhu et al., "Plantamajoside attenuates isoproterenol-induced cardiac hypertrophy associated with the HDAC2 and AKT/GSK-3 $\beta$ signaling pathway," ChemicoBiological Interactions, vol. 307, pp. 21-28, 2019.

[32] T. F. Tzeng, W. Y. Liu, S. S. Liou, T. Y. Hong, and I. M. Liu, "Antioxidant-rich extract from Plantaginis semen ameliorates diabetic retinal injury in A streptozotocin-induced diabetic rat model," Nutrients, vol. 8, 2016. 